\title{
Irritable bowel syndrome, gastro-oesophageal reflux, and bronchial hyper-responsiveness in the general population
}

\author{
T M Kennedy, R H Jones, A P S Hungin, H O’Flanagan, P Kelly
}

\begin{abstract}
Background-Associations have been shown between irritable bowel syndrome (IBS) and gastro-oesophageal reflux, between gastro-oesophageal reflux and asthma, and more recently between IBS and bronchial hyper-responsiveness (BHR).
\end{abstract}

Aims-To explore the inter-relations between these conditions.

Subjects-A randomly selected community sample of 4432 adults.

Methods-A validated postal symptom questionnaire investigating the associations between IBS, gastro-oesophageal reflux symptoms, and symptomatic BHR. Results-3169 questionnaires $(71.7 \%$ response) returned by 1451 men and 1718 women were analysed. One year prevalences, in men and women respectively, of IBS were $10.5 \%$ and $22.9 \%$, of dyspepsia $26.3 \%$ and $25.25 \%$, of gastro-oesophageal reflux symptoms $29.4 \%$ and $28.2 \%$, of BHR $13.2 \%$ and $14.6 \%$, and of chronic bronchitis $8.3 \%$ and $4.9 \%$. Logistic regression showed independent associations between IBS and BHR, gastro-oesophageal reflux symptoms, and dyspepsia. There was no significant independent association between IBS and chronic bronchitis. In men and women the odds ratio with $95 \%$ confidence interval (CI) for IBS and gastro-oesophageal reflux symptoms was $2.6(2.1-3.1 ; p<0.001)$ and for IBS and BHR 2.1 (1.7-2.7; p<0.001). These associations held on stratifying for sex and consultation behaviour. IBS, gastro-oesophageal reflux symptoms, and bronchial hyper-responsiveness occurred more frequently together than expected, $2.5 \%$ (95\% CI 2.41-2.57) of the sample having all three conditions compared with an expected prevalence of $0.7 \% \quad(95 \%$ CI $0.66-0.71)$. The conditions were independently associated with each other.

Conclusions-These observations may indicate the presence of an underlying disorder producing symptoms in gastrointestinal and respiratory systems. (Gut 1998;43:770-774)

Keywords: irritable bowel syndrome; asthma; dyspepsia; gastro-oesophageal reflux; epidemiology

Symptoms of irritable bowel syndrome (IBS) ${ }^{1}$ and gastro-oesophageal reflux ${ }^{2}$ are common in the general population but only a minority of people with these symptoms consult a doctor about them. ${ }^{34}$ The two conditions often coexist ${ }^{1}$ and it has been proposed that in many patients gastro-oesophageal reflux and IBS may share a common aetiology. ${ }^{5}$

There have also been reports of an unquantified association between gastro-oesophageal reflux and asthma ${ }^{6}$; however these studies have been carried out on small study samples not representative of patients in the general population with these conditions. Explanations proposed for the association have been limited to theories of direct causation stating that asthma may cause gastro-oesophageal reflux by raised intrathoracic pressure, ${ }^{7}$ or that gastrooesophageal reflux may cause asthma by aspiration $^{7}$ or by a vagally mediated reflex. ${ }^{8}$ The possibility of an underlying disorder which produces both gastro-oesophageal reflux and asthma has not been explored.

White et al have shown an excess prevalence of bronchial hyper-responsiveness (BHR) among patients with IBS. ${ }^{9}$ BHR is defined as excessive airway constriction in response to an inhaled stimulus. ${ }^{10}$ It is a ubiquitous finding in asthma and has been incorporated into recent definitions. ${ }^{11}$ The finding of White et al raises the possibility of an underlying disorder which produces both asthma and IBS. Toelle et al have proposed that a suitable epidemiological definition for asthma would be the presence of BHR in association with asthma symptoms ${ }^{12}$ and a postal questionnaire which identifies symptomatic BHR has been created. ${ }^{13}$

We were interested in determining the associations between IBS, gastro-oesophageal reflux, and asthma but wished to do so in a study free from referral bias and large enough to have adequate statistical power. We sent a postal questionnaire to a large sample of adults chosen at random from the general population and found that the three conditions of IBS, gastro-oesophageal reflux symptoms, and symptomatic BHR are associated.

\section{Materials and methods}

As the Rome criteria ${ }^{14}$ have not been validated we defined IBS as abdominal pain on more than six occasions in the previous year along with three or more of the Manning criteria. ${ }^{15}$ These are reasonably specific requirements as Manning reported satisfactory specificity with abdominal pain and two or more of the Manning criteria. We defined dyspepsia as the presence of upper abdominal discomfort on more than six occasions during the previous year, or excess upper abdominal fullness after eating or drinking, occurring on more than six occasions during the previous year. ${ }^{2}$ 
There is confusion regarding the clinical use of the term gastro-oesophageal reflux disease (GORD). Presently the term covers a broad range of clinical states including asymptomatic gastro-oesophageal reflux identified by oesophageal $\mathrm{pH}$ study, symptomatic gastrooesophageal reflux, gastro-oesophageal reflux associated with pathological change in the oesophagus (for example, oesophagitis) and patients with severe symptoms, normal endoscopy, and abnormal pH metry ${ }^{16}$ (endoscopy negative GORD). We propose that "gastrooesophageal reflux" be used to describe the reflux of gastric contents into the oesophagus, that heartburn and acid regurgitation are "gastro-oesophageal reflux symptoms", and that the term GORD be reserved for patients with oesophageal pathology (for example, oesophagitis) thought to be due to gastrooesophageal reflux, and those with endoscopy negative GORD.

We identified patients with gastrooesophageal reflux symptoms if they had either heartburn or acid regurgitation on more than six occasions during the previous year. These symptoms have been shown reliably to identify patients with gastro-oesophageal reflux (on $\mathrm{pH}$ testing) when they dominate the symptom spectrum $^{17}$ but have also been shown frequently to coexist with other upper abdominal symptoms. ${ }^{2} 18$

The BHR questionnaire has been validated against a histamine inhalation challenge test in a large UK population sample aged 18 to 64 years. ${ }^{13}$ The following questions were most relevant in identifying patients with symptomatic BHR (sensitivities and specificities in brackets): experiencing an asthma attack in the previous year $(0.22,0.99)$, and waking in the night short of breath during the previous year $(0.53$, $0.90)$; a report of being on treatment for asthma is considered to be highly specific for BHR but not very sensitive. ${ }^{19}$ On the recommendations of the team which developed the questionnaire we identified symptomatic BHR in patients who reported being on asthma medication or who had experienced an asthma attack in the previous 12 months or woke during the night short of breath in the previous 12 months.

Nocturnal dyspnoea may not be as specific for BHR among elderly subjects as there may be overlap with cardiac symptoms. In addition tobacco smoking may be increasingly important in the aetiology of BHR among people over 40 years of age $\mathrm{an}^{20}$ and symptomatic BHR may be less specific for asthma in that age group. We therefore carried out a separate backward stepwise logistic regression to investigate the associations between IBS, gastrooesophageal reflux symptoms, dyspepsia, and BHR in people aged under 40.

It has been proposed that patients with IBS may display learned illness behaviour ${ }^{21}$ and may be expected to complain of a diverse range of symptoms without organic cause. We controlled for this in two ways: by carrying out a backward stepwise logistic regression to check whether there was an independent association between IBS and chronic bronchi- tis, there being no a priori reason to suspect an independent association between these two conditions; and by determining the effect of consultation behaviour on the association between IBS and BHR. Chronic bronchitis was defined as the presence of bronchial hypersecretion and identified using the UK MRC Respiratory Symptoms Questionnaire. ${ }^{22}$

We constructed a symptom questionnaire from individual questionnaires on IBS, ${ }^{1}$ dyspepsia, ${ }^{23} \mathrm{BHR},{ }^{13}$ and the UK MRC questionnaire for chronic bronchitis. ${ }^{22}{ }^{24}$ These have been validated in community populations. The composite questionnaire was piloted in a sample of 100 people aged 20 to 69 years, selected from a general practice register. The study population was stratified into 10 year age bands prior to random sampling. The questionnaire was not changed as a result of this pilot. Ethical approval was granted by local ethics committees. Return of a completed questionnaire was taken as consent to participate. Assuming prevalences of $12 \%$ and $20 \%$ for BHR and IBS respectively and a response of $70 \%$, a power calculation ( $80 \%$ power, $5 \%$ level of significance) indicated that we required a sample of 4500 people to detect an odds ratio of 2 or more for an association between IBS and BHR. Odds ratios rather than relative risks were determined as we were conducting a cross sectional study investigating association not causation. We selected people from the practice registers of six general practices which are members of the Northern Region Research Network (NoReN). Prior to randomisation we stratified the study population into 10 year age bands from 20 to 69 years of age inclusive. In two practices we assigned consecutive numbers to each person on the registers and selected a 1:10 sample from each 10 year stratum using random number tables. In four practices we used a software facility on the practice computer to generate a random sample. This method had been used in a previous study on dyspepsia and was found to generate a sample of patients whose demographic characteristics were representative of those of the geographical area in which the practice was situated.

A covering letter on each practice's letterhead described the study and was signed by a general practitioner from the patient's practice and by one of the research team. General practitioners were given a list of selected patients for approval before mailing. Each subject received the questionnaire, the covering letter, and a reply paid envelope. The address on the outgoing envelope and covering letter were personalised. We included a contact address and phone number and provided an answerphone facility for queries outside office hours. Two reminders at one month intervals enclosed a questionnaire, reply paid envelope, and covering letter. Sample details were checked prior to mailing to avoid sending a questionnaire to someone who had become seriously ill or died during the study period.

Data were double entered on computer and analysed using Epi Info and SPSS software. Prevalences were derived with 95\% confidence intervals. Associations were analysed by $\chi^{2}$ test. 
Table 1 Logistic regression of covariates predicting the presence of IBS

\begin{tabular}{|c|c|c|c|c|}
\hline & \multicolumn{2}{|c|}{ All subjects } & \multicolumn{2}{|c|}{ Subjects aged $<40$} \\
\hline & $\beta$ & Significance & $\beta$ & Significance \\
\hline Bronchial hyper-responsiveness & -0.50 & $<0.001$ & -0.55 & $<0.005$ \\
\hline Bronchitis $\star$ & -0.01 & $>0.9$ & & \\
\hline Gastro-oesophageal reflux symptoms & -0.30 & $<0.01$ & -0.35 & $<0.05$ \\
\hline Dyspepsia & -1.55 & $<0.001$ & -1.38 & $<0.001$ \\
\hline
\end{tabular}

${ }^{\star}$ Bronchitis was not independently associated with IBS at a significant level.

Odds ratios were determined with $95 \%$ confidence limits. We carried out backward stepwise logistic regressions to identify which clinical syndromes predicted the presence of IBS.

\section{Results}

We selected 4476 subjects; 38 were excluded at their general practitioner's request, due to concurrent illness. Six people died during the study, leaving 4432 subjects. The postal service identified $224(5 \%)$ people as no longer resident at the recorded address and returned their letters. We received 3179 questionnaires ( $71.7 \%$ response). Non-responders were more likely to be male $(\mathrm{p}<0.01)$ and to be younger than responders. The mean age of nonresponders was 38 years, and of responders was 42 years $(\mathrm{p}<0.001)$. Compared to national UK figures $^{25}$ responders are slightly underrepresentative of males and females aged 20 to 29 years with respective sample prevalences expressed as a percentage of responders aged 20 to 64 years of $20.5 \%$ (95\% confidence interval (CI) $15.8-25.2)$ and $21.1 \%$ (95\% CI 16.8-25.4). Males and females aged 45 to 59 years were over-represented with respective prevalences of $34.6 \%(30.3-39.9)$ and $33.6 \%$ (29.6-37.6). Ten inadequately completed questionnaires were excluded. Data from 3169 questionnaires were double entered on computer, representing 1451 men and 1718 women.

The twelve month prevalences of IBS (with $95 \% \mathrm{CI}$ for men and women respectively) were $10.5 \%(8.9-12.1)$ and $22.9 \%(20.9-24.9)$, of dyspepsia $26.3 \%(25.3-27.3)$ and $26.25 \%$ (25.3-27.3), and of gastro-oesophageal reflux symptoms $29.4 \% \quad(27.1-31.7)$ and $28.2 \%$ (26.1-30.3). The prevalence of BHR for men and women was respectively $13.2 \%$ (11.5-14.9) and $14.6 \%(12.9-16.3)$ and of chronic bronchitis was $8.3 \%(6.9-9.7)$ and $4.9 \%(3.7-5.9)$.

IBS and BHR occurred together more commonly than expected and this association held when the data were stratified for the presence of gastro-oesophageal reflux symptoms, producing a Mantel-Haenzel (M-H) stratified odds ratio (OR) of $1.84 \quad(95 \%$ CI $1.45<\mathrm{OR}<2.34) .{ }^{26}$ Likewise the association between gastro-oesophageal reflux symptoms and BHR held when stratified for IBS: crude odds ratio $=2.25, \mathrm{M}-\mathrm{H}=2.05(95 \% \mathrm{CI}$

Table 2 Associations between the symptom complexes expressed as odds ratios (95\% confidence limits)

\begin{tabular}{llll}
\hline & IBS & GOR symptoms & Dyspepsia \\
\hline BHR & $2.1(1.67-2.71)$ & $2.25(1.81-2.78)$ & $2.04(1.64-2.54)$ \\
Dyspepsia & $5.5(4.50-6.73)$ & $6.49(5.42-7.76)$ & \\
GOR symptoms & $2.6(2.14-3.17)$ & & \\
\hline
\end{tabular}

GOR, gastro-oesophageal reflux.
$1.66<\mathrm{OR}<2.54)$. These results show that IBS, gastro-oesophageal reflux symptoms, and BHR are independently associated with each other.

Backward stepwise logistic regression also showed independent associations between IBS and dyspepsia, gastro-oesophageal reflux symptoms, and BHR (table 1). There was no independent association with chronic bronchitis. A similar regression among people under the age of 40 (not including chronic bronchitis as there is insufficient study power to investigate that association among people aged less than 40 years) has been carried out. Table 2 reports on the individual associations between any two symptom complexes among those conditions shown to be independently associated with IBS on logistic regression.

We also determined whether subjects who are on treatment for asthma (diagnosed asthmatics) are more likely to suffer symptoms of IBS and found that having asthma was associated with IBS; OR $=1.57(1.15<\mathrm{OR}<2.14)$.

Subjects with IBS were more likely to experience dyspepsia than were controls; $57.5 \%$ of subjects with IBS had dyspepsia, compared with $19.7 \%$ of those without. Of those with IBS, $46.5 \%$ had gastro-oesophageal reflux symptoms while $25.0 \%$ of those without IBS had gastro-oesophageal reflux symptoms. Regarding IBS and $\mathrm{BHR}, 23 \%$ of people with IBS compared with $12 \%$ of controls had symptoms of BHR. Stratified analysis showed that sex did not influence the associations between IBS and gastro-oesophageal reflux (M-H odds ratio $=2.72(2.24-3.30)$ ), or between IBS and $\mathrm{BHR}(\mathrm{M}-\mathrm{H}$ odds ratio $=2.11(1.67-2.67))$.

Figure 1 illustrates the relation between IBS, gastro-oesophageal reflux symptoms, and BHR. All three symptom complexes were observed to coexist in $2.5 \%$ of the sample compared with an expected prevalence of $0.68 \%$ if one assumed that no association existed between them. When the 95\% confidence intervals for the observed and expected prevalences are calculated they do not overlap, indicating that the three conditions occur together more commonly than expected at the $5 \%$ level of significance. These associations held on stratifying for sex (table 3 ).

We compared people with IBS who had consulted their general practitioner with those who had not and found that of 546 people with IBS, $109(20 \%)$ had consulted their general practitioner regarding at least one IBS symptom in the previous two years. Of those IBS consulters $43.1 \%$ (95\% CI 38.5-47.7) had gastro-oesophageal reflux symptoms and $22.0 \%$ (95\% CI 18.6-25.4) had BHR. Of people with IBS who had not consulted, 
Table 3 Co-existence of IBS, gastro-oesophageal reflux symptoms and BHR

\begin{tabular}{lll}
\hline & $\begin{array}{l}\text { Observed prevalence } \\
(95 \% \text { CI })\end{array}$ & $\begin{array}{l}\text { Expected prevalence } \\
(95 \% \text { CI })\end{array}$ \\
\hline Male & $1.65 \%(1.57-1.73)$ & $0.41 \%(0.39-0.43)$ \\
Female & $3.20 \%(3.05-3.35)$ & $0.94 \%(0.90-0.98)$ \\
Total & $2.49 \%(2.41-2.57)$ & $0.68 \%(0.66-0.71)$ \\
\hline
\end{tabular}

CI, confidence interval.

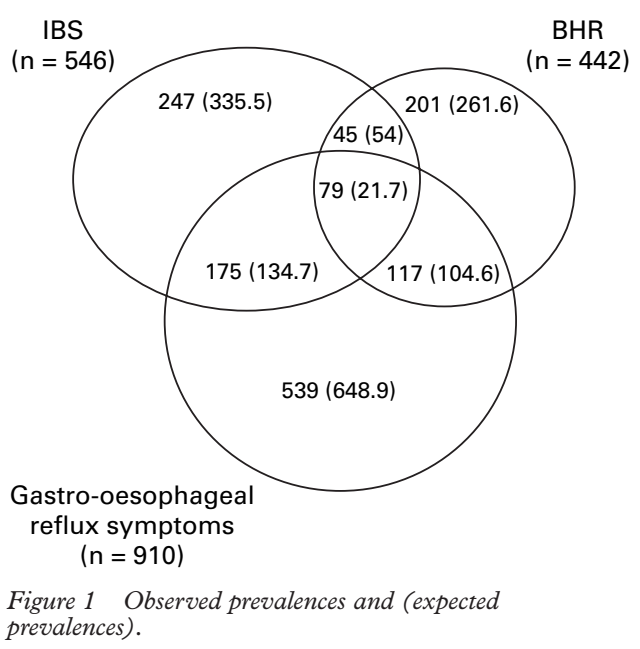

$47.4 \%$ (95\% CI 45.9-48.9) had gastrooesophageal reflux symptoms and $22.9 \%$ (95\% CI 21.4-24.7) had symptoms of BHR. The confidence intervals for the prevalences overlap, indicating that associations between IBS and gastro-oesophageal reflux and between IBS and BHR were the same whether or not people with IBS had consulted their general practitioner in the previous two years; consultation behaviour therefore did not affect the association.

\section{Discussion}

The methodology used in this study was robust. General practice registers in the UK offer the best means of sampling the general population. ${ }^{27}$ Registration is almost complete for the population and address details are accurately maintained and regularly updated. The overall questionnaire response was good at $71.7 \%$ but younger age and male sex were indicators of lower response. Non-responders were not followed up as ethical consent required return of the questionnaire to indicate consent for participation in the study. Although it is possible that a "personal significance bias" influenced subjects' likelihood to return the questionnaire, there is evidence that most biases disappear when a response of $70 \%$ or more is achieved. ${ }^{28}$

We investigated the associations between symptom complexes as it would be impractical to carry out methacholine challenge testing and gastrointestinal endoscopy on 3200 subjects. We are aware that not all subjects with the symptoms described will have the underlying target condition but this was a hypothesis generating exercise. As well as avoiding the referral bias inherent in secondary care based surveys of this kind, the sample size chosen was sufficiently large to show significant associations between the selected symptom groups even among patients aged less than 40 where the specificity of the BHR questionnaire is likely to be at its highest.

We have shown that IBS, gastro-oesophageal reflux symptoms, and symptomatic BHR occur more frequently together than expected and that the conditions are independently associated with each other. The absence of an independent association between IBS and chronic bronchitis was reassuring (as well as informative) in showing that the questionnaire we used was unlikely to have produced a positive response bias for the presence of respiratory symptoms in the study subjects. This, coupled with the fact that we have shown that the associations are uninfluenced by consultation behaviour would indicate that hypervigilance and a tendency for increased symptom reporting is unlikely to account for the results.

These associations are difficult to explain because two physiological systems are involved. One possible explanation is that the gastrointestinal and respiratory symptoms in our subjects are caused by a common (but as yet unidentified) underlying disorder, capable of producing symptoms in more than one physiological system and resulting in an indirect association between seemingly disparate conditions. A number of candidate mechanisms exist for this: one is a generalised disorder of bronchial, gastrointestinal, and other smooth muscle. ${ }^{2}$ Another is a more complex neuromuscular (afferent or efferent) disorder with altered visceral sensitivity and shared transmitter dysfunction. The third is the possibility of an inflammatory aetiology for the respiratory and gastrointestinal symptoms. ${ }^{29}$ For the reasons mentioned it is unlikely that the associations are a product of a cognitive tendency to over report among patients with IBS.

This cross sectional survey is incapable of determining the cause of the strong associations which we have shown between symptom groupings. The next research steps may be to undertake studies in subjects selected from our study population to determine the extent to which symptomatic associations are mirrored in physiological dysfunction. This would help in further clarifying the reporting tendencies of these patients as well as investigating possible physiological reasons for these associations.

We thank the patients, doctors, and staff of Eaglescliffe Medical Practice, Thornaby and Ingleby Barwick Medical Group, Skelton Group Practice, Tennant Street Surgery, Moorland's Surgery, and Sedgefield Group Practice; also Dr Kevin Jones and Clare Tait. This study was supported in part by grants from the Scientific Foundation Board of the Royal College of General Practitioners, the Research and Development Directorate of the Northern Regional Health Authority, the Cleveland Primary Care Research Panel, and the Primary Care Society for Gastroenterology. Tom Kennedy held a Regional Research Training Fellowship during part of this study and was attached to the Department of Primary Health Care, University of Newcastleupon-Tyne.

1 Jones R, Lydeard S. Irritable bowel syndrome in the general population. BMF 1992;304:87-90.

2 Jones R, Lydeard S. Prevalence of symptoms of dyspepsia in the community. BMF 1989;298:30-2.

the community. BMF 1989;298:30-2.
3 Kettell J, Jones R, Lydeard S. Reasons for consultation in irritable bowel syndrome; symptoms and patients characteristics. Br F Gen Pract 1992;42:459-61. 
4 Jones RH, Lydeard SE, Hobbs EDR. Dyspepsia in England and Scotland. Gut 1990;31:401-5.

Smart HL Nicholson DA, Atkinson M. Gastro-oesophageal reflux in the irritable bowel syndrome. Gut 1986;27:122731.

6 Mays EE. Intrinsic asthma in adults, association with gastro-oesophageal reflux. $\mathcal{F A M A}$ 1976;236:2626-8.

7 Boyle JT, Tuchman DN, Altschuler SM. Mechanisms for the association of gastro-oesophageal reflux and bronchospasm. Am Rev Respir Dis 1985;131(suppl):S16-20.

8 Mansfield LE, Stein MR. Gastro-oesophageal reflux and asthma: a possible reflex mechanism. Ann Allergy 1978;41: 224-6.

9 White AM, Stephens WH, Upton AR, et al. Airway responsiveness to inhaled methacholine in patients with irritable bowel syndrome. Gastroenterology 1991;100:68-74.

10 Snashel PD, Pauwels R. Definitions and historical perspective. In: Nadel JA, Pauwels R, Snashel PD, eds. Bronchial hyperresponsiveness. London: Blackwell Scientific Publications, 1992 .

11 American Thoracic Society. Definitions and classification of chronic bronchitis, asthma and pulmonary emphysema. Am Rev Respir Dis 1962;85:762-8.

12 Toelle BG, Peat JK, Salome CM, et al. Toward a definition of asthma for epidemiology. Am Rev Respir Dis 1992;146 633-7

13 Burney PG, Chinn S, Britton JR, et al. What symptoms predict the bronchial response to histamine? Evaluation in a community survey of the bronchial symptoms questionnaire (1984) of the International Union Against Tuberculosis and Lung Disease. Int F Epidemiol 189;18:165-73.

14 Drossman DA, Thompson WG, Talley NJ, et al. Identification of subgroups of functional gastrointestinal disorders. Gastroenterology International 1990;3:159-72.

15 Manning AP, Thompson WG, Heaton KW, et al. Towards positive diagnosis of the irritable bowel. BMF 1978;2:653-4

16 Schindlbeck NE, Ippisch H, Klauser AG, et al. Which pH threshold is best in oesophageal pH monitoring. Am $\mathcal{f}$ Gas troenterol 1991;86:1138-41.
17 Klauser AG, Schindlbeck NE, Muller-Lissner SA. Symptoms in gastro-oesophageal reflux disease. Lancet 1990; 335:205-8.

18 Talley N, Weaver AL, Tesmer DL, et al. Lack of discriminant value of dyspepsia subgroups in patients referred for upper endoscopy. Gastroenterology 1993;105:1378-86.

19 Burney PGJ, Chinn S. Developing a new questionnaire for measuring the prevalence and distribution of asthma. Chest 1987;91(suppl):79-83S.

20 Burney PGJ, Britton JR, Chinn S, et al. Descriptive epidemiology of bronchial reactivity in an adult population: results from a community study. Thorax $1987 ; 42: 38-44$.

21 Whitehead WE, Wriget C, Fedoravicius AS, et al. Learned illness behaviour in patients with irritable bowel syndrome and peptic ulcer. Dig Dis Sci 1982;27:202-8.

22 Medical Research Council. Definition and classification of chronic bronchitis for clinical and epidemiological purposes Lancet 1965 ; i: $775-9$.

23 Kennedy T, Jones R. Development of a postal health status questionnaire to identify people with dyspepsia in the genquestionnaire to identify people with dyspepsia in the gen-

24 Fletcher CM, Peto R, Tinker CM. A comparison of the assessment of simple bronchitis (chronic mucus hypersecretion) by measurements of sputum volume and by standardised questions on phlegm production. Int $\mathcal{F}$ Epidemiol 1974;3:315.

25 Wisniewski D (ed). Annual abstracts of statistics 1997, number 133. London: HMS Publications, 1997

26 Fleiss JL. Statistical methods for rates and proportions. 2nd edn. New York: Wiley, 1981.

27 Fleming DM. Morbidity registration and the fourth general practice morbidity survey in England and Wales. Scand 7 Prim Health Care 1993; (suppl 2):37-41.

28 Goudy WJ. Interim response to a mail questionnaire: impacts on variable relationships. Iowa Agriculture and Home Economics Experiment Station. Journal Paper No. J8456. Iowa State University, 1976:13.

29 Collins SM. Is the irritable gut an inflamed gut? Scand 7 Gastroenterol 1992;27(suppl 192):102-5. 